\title{
Kernos
}

Revue internationale et pluridisciplinaire de religion grecque antique

$31 \mid 2018$

Varia

\section{Les grains du sacrifice. Le lancer d'orges dans la pratique sacrificielle en Grèce ancienne}

\section{Stéphanie Paul}

\section{(2) OpenEdition \\ Journals}

\section{Édition électronique}

URL : http://journals.openedition.org/kernos/2673

DOI : 10.4000/kernos.2673

ISSN : 2034-7871

\section{Éditeur}

Centre international d'étude de la religion grecque antique

\section{Édition imprimée}

Date de publication : 1 décembre 2018

Pagination : 59-72

ISBN : 978-2-87562-055-2

ISSN : 0776-3824

\section{Référence électronique}

Stéphanie Paul, «Les grains du sacrifice. Le lancer d'orges dans la pratique sacrificielle en Grèce ancienne », Kernos [En ligne], 31 | 2018, mis en ligne le 01 octobre 2020, consulté le 25 janvier 2021. URL : http://journals.openedition.org/kernos/2673 ; DOI : https://doi.org/10.4000/kernos.2673

Ce document a été généré automatiquement le 25 janvier 2021. 


\title{
Les grains du sacrifice. Le lancer d'orges dans la pratique sacrificielle en Grèce ancienne
}

\author{
Stéphanie Paul
}

Cet article est issu d'une communication présentée à une journée d'étude organisée le 17 mai 2013 à l'Université de Paris-Sorbonne. Qu'il me soit permis de remercier ici chaleureusement Magali De Haro Sanchez et Véronique Boudon-Millot de leur invitation à prendre part à cette journée. Je dois également ma reconnaissance à Jan-Mathieu Carbon et Vinciane PirenneDelforge, pour leurs commentaires toujours précieux. Abréviations utilisées : CGRN-Collection of Greek Ritual Norms (http://cgrn.ulg.ac.be); LSAM - F. SOKOLOWSKI, Lois sacrées de l'Asie mineure, Paris, 1955 ; LSCG - ID., Lois sacrées des cités grecques, 1969 ; LSS - ID., Lois sacrées des cités grecques. Supplément, 1962. Sauf mention contraire, les dates s'entendent avant notre ère.

Dans le foisonnement d'études consacrées au sacrifice grec, les céréales n'ont attiré l'attention que de manière marginale ${ }^{1}$. Pourtant, au même titre que le vin, répandu en libations, celles-ci font partie intégrante de la pratique sacrificielle. De l'offrande de grains à la préparation de gâteaux, leur utilisation fait preuve d'une grande diversité dont témoignent, notamment, les textes épigraphiques. Parmi ces différentes utilisations, il en est une dont la signification a davantage intrigué les commentateurs : le lancer de grains d'orge en prélude à la mise à mort de l'animal.

2 Le geste a tout particulièrement intéressé l'historiographie allemande du tournant du $\mathrm{xx}^{\mathrm{e}}$ siècle, où se croisent diverses tendances interprétatives. Ainsi, H. von Fritze, en reprenant les commentaires des anciens, le considérait comme un reliquat d'une ancienne forme de sacrifice en relation avec la première étape de la préparation du pain $^{2}$. Se distançant de son prédécesseur, L. Ziehen donnait au lancer d'orges une fonction cathartique, en se fondant sur l'association récurrente de la corbeille sacrificielle (kanoun) et du vase contenant l'eau pour les lustrations (chernips) ${ }^{3}$. À son tour, P. Stengel y voyait, outre cette interprétation cathartique - qu'il considérait toutefois comme plus tardive - une offrande à la déesse Gè, génitrice de la vie céréalière ${ }^{4}$. Les diverses explications proposées ont souvent été subordonnées aux 
modèles interprétatifs du sacrifice grec en général. Ainsi, dans la lignée des thèses développées en 1979 dans La Cuisine du sacrifice, qui plaçait la consommation des viandes au cœur de l'interprétation, les grains d'orge ont été perçus, de même que le vin, comme une référence au fondement de l'alimentation humaine ${ }^{5}$. Par ailleurs, en accord avec une autre thèse qui mettait en évidence la violence à l'œuvre dans les rites sacrificiels, le geste aurait été revêtu d'une nature agressive, symbolisant ainsi le refus d'une nourriture primitive qui n'était désormais plus consommée comme telle ${ }^{6}$.

La présente étude propose de faire le point sur cette question en reprenant le dossier des sources littéraires, afin de réévaluer la pertinence de certaines de ces interprétations. Dans un second temps, on parcourra la documentation épigraphique, plus rarement invoquée à l'appui de cette question, mais qui a l'avantage d'offrir une vision plus complexe des pratiques sacrificielles par la mise en évidence des variations et particularités locales.

\section{D’Homère aux lexicographes : parcours des sources littéraires}

Dans l'épopée homérique, la pratique du lancer d'orges est exprimée par le terme

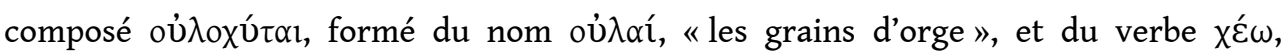
«verser $»^{7}$. À l'exception d'une occurrence, le mot intervient toujours dans le cadre d'un sacrifice sanglant et rend compte d'un geste antérieur à la mise à mort de l'animal, accompagnant la prière adressée à la divinité 8 . Aussi, l'enchaînement d'actions est toujours le même : les participants au sacrifice prennent les grains en main (oủ $\lambda o x u ́ \tau \alpha \varsigma$

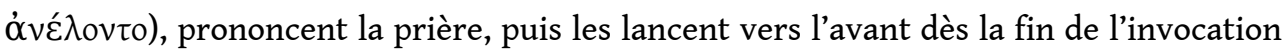

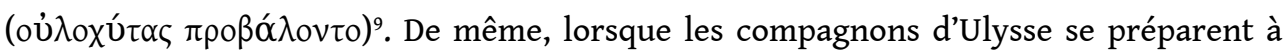
sacrifier les vaches d'Hélios dans l'odyssée, ils se voient contraints d'utiliser des feuilles de chêne à défaut de grains d'orge, et c'est uniquement après s'être procurés ce substitut que la prière peut être prononcée ${ }^{10}$. La même association est encore évidente dans un autre passage de l'Odyssée, lorsque Pénélope adresse, sur le conseil de sa nourrice, une prière à Athéna afin d'implorer sa protection envers Télémaque, parti à la recherche de son père :

Ayant séché ses pleurs et baigné son visage, Pénélope, vêtue d'une robe sans tache, regagna son étage avec ses chambrières et remplit sa corbeille des orges de

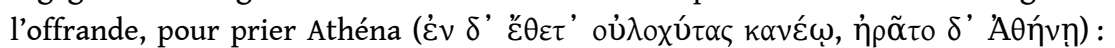
«Fille du Zeus qui tient l'égide, Atrytonée, exauce ma prière ! ah! si dans ce manoir Ulysse l'avisé t'a jamais fait brûler la graisse et les cuisseaux d'un bœuf ou d'un mouton, l'heure est enfin venue pour moi qu'il t'en souvienne ! ... ah! sauve-moi mon fils! déjoue, des prétendants, la criminelle audace!» Elle dit et poussa les clameurs rituelles; la déesse entendit son imprécation ${ }^{11}$.

5 L'absence dans ce passage de sacrifice animal, auquel les oulochytai sont habituellement associés, a suscité la perplexité des commentateurs. On a considéré que les orges étaient revêtues, au même titre que les ablutions, d'une fonction cathartique ${ }^{12}$. Toutefois, la simultanéité de ces deux actions n'implique pas de facto une même signification, et l'interprétation "cathartique » du lancer d'orges ne trouve guère de fondement par ailleurs dans les sources antiques. D'autres y ont vu une simple offrande de grains ${ }^{13}$, mais l'emploi du terme oulochytai, référence manifeste 
au lancer d'orges, reste alors inexpliqué. Or, si l'on s'attache au contenu de la prière, le sens du passage s'éclaire. En effet, la reine souhaite rappeler à Athéna les sacrifices sanglants autrefois accomplis par Ulysse à son égard afin que, en souvenir des offrandes reçues par le passé, elle exerce une nouvelle fois sa bienveillance. Le geste consistant à placer les oulochytai dans le kanoun, qui évoque immanquablement le sacrifice animal, viserait donc à redoubler l'énoncé de la prière, en activant chez la déesse, de manière plus concrète encore, le souvenir de ces offrandes. Par un procédé similaire, l'association récurrente du kanoun et de la chernips dans les sources littéraires et iconographiques en est venue, par une sorte de synecdoque, à résumer l'ensemble de la procédure ${ }^{14}$.

7 Si le terme oulochytai n'apparaît plus guère par la suite qu'en référence à, ou en imitation de l'épopée homérique ${ }^{15}$, le lancer d'orges est bien attesté dans la littérature postérieure. C'est une comédie d'Aristophane qui dépeint le tableau le plus animé de la phase préliminaire du sacrifice. Trygée donne des ordres à son serviteur pour offrir un sacrifice à la Paix, et le charge de présenter les orges ( $\pi \rho$ ó $\varepsilon i v \varepsilon \tau \tilde{\omega} v$ ỏ $\lambda \tilde{\omega} v)$ et d'en jeter

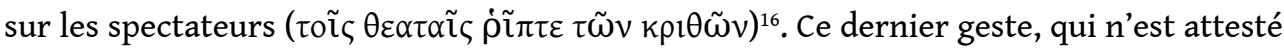
nulle part ailleurs, pourrait être en réalité une exagération du poète à visée humoristique. L'allusion est d'autant plus suspecte qu'elle ouvre la voie à une plaisanterie grivoise jouant sur l'homonymie de $\kappa \rho \imath \theta$, à la fois l'orge et le membre viril ${ }^{17}$.

Du côté de la tragédie, Euripide est le seul à faire allusion au lancer d'orges en ouverture du sacrifice. Dans l'Électre, Égisthe lance les grains d'orge - désignés ici par le terme $\pi \rho 0 \chi \cup ́ \tau \alpha 1$ - en direction de l'autel, tout en prononçant une prière ${ }^{18}$. Dans l'Iphigénie à Aulis, les grains d'orge sont destinés à être lancés des deux mains plus précisément vers le feu purificateur de l'autel. Plus loin, les grains eux-mêmes sont qualifiés de " purificateurs ", lorsqu'ils se consument à la flamme de l'autel.

Davantage qu'un argument en faveur d'une fonction cathartique des orges, il faudrait plutôt y voir une hypallage : tout en s'accordant, pour la syntaxe, avec $\pi \rho 0 x u ́ \tau \alpha \iota \varsigma, ~ c ' e s t$ au feu ( $\pi \tilde{\mathrm{V}} \rho$ ) que l'adjectif $\kappa \alpha \theta \alpha \rho \sigma i ́ o l \sigma l$ se rapporte pour le sens ${ }^{19}$. Ce procédé ne vise qu'à dramatiser davantage ce qui est en réalité non pas un sacrifice animal, mais humain. On constate ainsi que la description du lancer d'orges est fortement soumise à la nature de la source. La tragédie, par son emphase sur la mise à mort de l'animal, aura tendance à passer sous silence les rites additionnels ou à les utiliser, comme c'est le cas chez Euripide, en vue d'accentuer la dramatisation du sacrifice. À l'inverse, la comédie en donne une impression plus légère, voire improvisée, et met plutôt l'accent sur les rites qui entourent la mise à mort et sur l'aspect alimentaire, à savoir le banquet. Tout en s'inscrivant dans un système de référence partagé des spectateurs, ces pièces comportent des exagérations dont il est parfois difficile de prendre la mesure ${ }^{20}$.

Même s'il n'est pas mentionné systématiquement dans toutes les descriptions, le lancer d'orges paraît être un élément constitutif et caractéristique du sacrifice grec. Ainsi, lorsqu'Hérodote définit en creux le rite sacrificiel chez les Perses, il constate que ce dernier ne comprend ni libations, ni aulos, ni bandelettes, ni grains d'orge (oủkì oủ même, Athénée évoque une particularité des rites sacrificiels des Béotiens, qui avaient l'habitude de sacrifier des anguilles à la manière des animaux sacrificiels, c'est-à-dire en les couronnant, en prononçant des prières et en jetant des grains d'orge (oủ $\lambda \alpha^{\prime} \varsigma \tau^{\prime}$ 
$\dot{\varepsilon} \pi ı \beta \alpha \lambda \lambda o v \tau \alpha \varsigma)^{22}$. L'auteur souligne ainsi le contraste entre offrande exceptionnelle et pratique habituelle, dont les orges font partie.

11 Les commentaires et les traductions modernes qui s'attachent à décrire le lancer de grains d'orge comportent deux éléments récurrents dans leur description qui paraissent absents des sources littéraires parcourues jusqu'ici: le fait que les grains auraient été, d'une part, mélangés avec du sel, et d'autre part, lancés en direction de l'animal sacrificiel. Dans la seconde hypothèse, le lancer de grains pourrait s'interpréter comme une consécration de l'animal, marquant son passage imminent de la sphère humaine à la sphère divine ${ }^{23}$. D'autres y ont vu en revanche une mise à distance de l'animal par rapport au groupe, par analogie avec le rituel du bouc émissaire ${ }^{24}$. Or, le seul indice qui pourrait appuyer l'idée que les grains étaient lancés sur l'animal est l'emploi du verbe غ̇đißó $\lambda \lambda \varepsilon ı v$ par Athénée dans le passage mentionné ci-dessus, et dont le préverbe pourrait suggérer que les grains étaient lancés sur les anguilles. Or Athénée a écrit son œuvre à Rome, dans le courant du $\mathrm{III}^{\mathrm{e}}$ siècle de notre ère, et bien qu'il tire son témoignage du grammairien alexandrin Agatharcide, il ne semble pas le citer textuellement. Plus tardivement encore, au v $v^{e}$ siècle de notre ère, Nonnos de Panopolis évoque la phase d'ouverture du sacrifice, lors de laquelle les

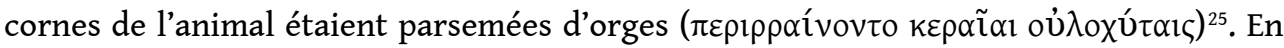
réalité, hormis le texte d'Euripide, selon lequel les orges étaient consumées à la flamme de l'autel, et le témoignage incertain d'Aristophane, c'est moins la destination des grains qui est mise en évidence que le mouvement du lancer, exprimé par le préverbe pro-, vers l'avant ${ }^{26}$. Ainsi, il est plausible que les grains n'aient pas été destinés à un endroit spécifique, mais atterrissaient à la fois sur l'autel, l'animal, et le sol.

Quant à l'idée que les grains auraient été mélangés à du sel ${ }^{27}$, seule une allusion difficile d'interprétation dans une comédie fragmentaire de Straton, auteur de la seconde moitié du IV siècle, pourrait y faire référence ${ }^{28}$. Or, le contexte n'est pas celui d'un sacrifice, mais de la préparation d'un repas par un cuisinier qui s'exprime en des termes homériques, provoquant force malentendus et incompréhensions de la part de son interlocuteur. Le sel y est mentionné parmi les ingrédients du repas, entre les oulochytai et la chernips, les deux éléments caractéristiques de la phase d'ouverture du sacrifice. Peut-être s'agirait-il d'une allusion à la présence du sel dans cette étape du rituel, mais l'interprétation est très incertaine, et le mélange d'ingrédients « sacrés » et «profanes » pourrait plus probablement renforcer l'effet humoristique de la scène.

13 En réalité, ces deux idées paraissent se fonder avant tout sur le témoignage des scholies et des lexicographes. Or, ces sources, de date incertaine, sont difficiles à manier pour l'interprétation des procédures sacrificielles, dans la mesure où elles proposent une compilation exhaustive et indistincte de définitions qui renvoient à des pratiques de contextes très divers. Le mélange d'orges et de sel, de même que le lancer de grains vers l'animal, sont récurrents dans plusieurs de ces témoignages, qui tirent dans la plupart des cas leur inspiration d'une même source ${ }^{29}$ :

Oulochytas: oulas. Ce sont des grains d'orge (kpı $\theta$ aí) mélangés à du sel, que l'on versait sur les animaux sacrificiels avant le sacrifice, soit en remerciement de l'abondance, ou en souvenir d'une nourriture ancestrale. Comme le dit Théophraste dans le Peri Heurèmatôn, avant que les hommes n'apprennent à moudre le fruit de Déméter, ils les mangeaient ainsi entiers. C'est ainsi qu'ils sont appelés oulai, comme le dit le poète (Scholia in Homeri Iliadem, I, 449). moins s'interroger, vu la date tardive de ces témoignages, sur l'éventualité d'une 
influence de l'immolatio romaine dans l'explication de la pratique grecque. En effet, lors de cette étape préliminaire à la mise à mort, la mola salsa, farine d'épeautre salée préparée par les Vestales, était répandue avec du vin sur la tête de l'animal dans un geste de consécration ${ }^{30}$. En revanche, l'ancienneté de l'orge qui est invoquée pour expliquer la symbolique du geste à plusieurs reprises parait être un topos évoqué par plusieurs auteurs antérieurs, dont Théophraste ${ }^{31}$.

\section{L'immuabilité d'une pratique à l'épreuve des inscriptions}

(1)

ure du sacrifice animal : on y trouve, en lieu du plus commun $k \rho \imath \theta \alpha i ́$, le terme oủ $\alpha$ í, qui y est étroitement attaché ${ }^{34}$. Un règlement d'Épidaure concernant la prothysis, sacrifice accompli avant l'incubation des patients dans le sanctuaire, demande au prêtre de fournir aux sacrifiants le matériel nécessaire moyennant remboursement : les grains (ó $\lambda \tilde{\alpha} v$ ), la bandelette et le bois pour le feu ${ }^{35}$. Dans deux ventes de prêtrise de Priène, c'est le prêtre ou la prêtresse qui fournit les grains (oủ $\lambda \alpha$ )́ en plus d'autres ingrédients nécessaires pour les sacrifices, gâteaux, parfums et encens ${ }^{36}$. À Myconos, ce sont les hiéropes qui sont chargés de fournir, sur les fonds sacrés, les animaux pour un sacrifice à Déméter, Korè et Zeus Bouleus, ainsi que du bois pour le feu et des grains (oủ $\lambda \alpha)^{37}$. De la même manière, à Cos, les hiéropes doivent prévoir une mesure de

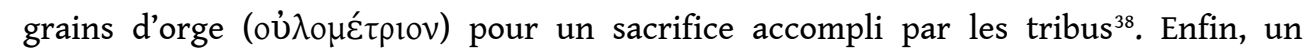
règlement de Lykosoura concernant le sanctuaire de Despoina établit la liste exhaustive du matériel nécessaire pour les rites préliminaires au sacrifice ( $\pi \rho \circ \sigma \theta u ́ \mu \alpha \tau \alpha)$, parmi lequel des branches d'olivier, du myrte, des rayons de miel et des grains d'orge que l'on

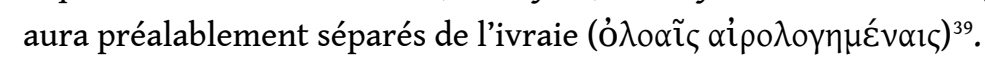

Dans ces inscriptions, les grains d'orge sont cités parmi le matériel à prévoir pour le sacrifice et ne font pas l'objet d'une réglementation particulière. Vu la tendance des sources épigraphiques à ne mentionner que le nécessaire ou ce qui s'écarte de la norme, il est tentant de conclure que la pratique du lancer d'orges allait de soi et qu'il était inutile de légiférer à son propos. Une célèbre inscription de Cos incite toutefois à s'interroger sur les potentialités de variation de cette pratique. Il s'agit d'une stèle appartenant au calendrier cultuel établi après le synécisme de la cité, qui rend compte en détail de l'organisation d'une fête en l'honneur de Zeus Polieus ${ }^{40}$. La veille d'un sacrifice bovin à Zeus prend place une série de rites préliminaires, parmi lesquels l'holocauste d'un porcelet. Après la proclamation de l'euphèmia, le texte prescrit de

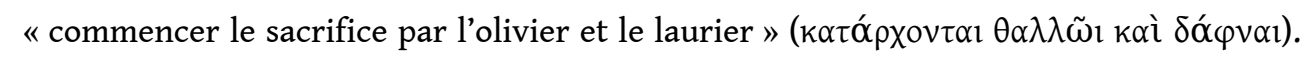
Or, le verbe $\kappa \alpha \tau \alpha \rho \chi \varepsilon ́ \sigma \theta \alpha l$ est, selon plusieurs textes littéraires, étroitement associé aux 
rites d'ouverture du sacrifice, et, dans l'épopée homérique, attaché de manière plus spécifique à l'aspersion d'eau lustrale et au lancer de grains d'orge ${ }^{41}$. Deux interprétations ont été proposées, se référant chacune à l'une de ces deux actions. Tandis que l'une y voit l'utilisation de branchages pour asperger d'eau l'animal

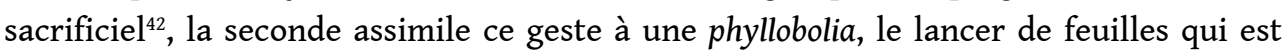
associé à la victoire d'un athlète, aux mariages ou aux funérailles ${ }^{43}$. Cette dernière idée ne manque pas d'évoquer le sacrifice des vaches d'Hélios dans l'Odyssée, pour lequel les compagnons d'Ulysse utilisent, à défaut de grains d'orge, des feuilles de chêne, et de l'eau pour les libations, au lieu du vin ${ }^{44}$. Le fait que les protagonistes, dépourvus des produits de la civilisation, en soient réduits à utiliser ce que la nature veut bien leur donner souligne ici le caractère interdit du sacrifice, qui aura des conséquences funestes ${ }^{45}$. Une telle interprétation ne peut, en revanche, valoir pour le cas de Cos. En effet, l'holocauste n'est pas un sacrifice «anormal», mais a pour fonction d'accompagner la proclamation, d'ouvrir le sacrifice du lendemain et d'accroître l'intensité de la fête par l'offrande supplémentaire d'un petit animal de moindre valeur $^{46}$. De plus, la symbolique revêtue par l'olivier est toute différente de celle du chêne dans l'odyssée, puisque, offert par Athéna, il s'agit d'un arbre faisant l'objet d'une culture par les hommes et par conséquent étroitement lié à la conception de la civilisation.

Par ailleurs, l'utilisation rituelle de branchages est bien représentée par la céramique ${ }^{47}$. Dans ce corpus, on peut signaler une amphore à figures noires du peintre de Berlin, qui représente l'arrivée d'une procession accompagnant le sacrifice d'un bœuf à Athéna. On y observe un personnage féminin, peut-être la prêtresse, en train de manipuler des rameaux de feuillage devant un autel, sans doute en un geste d'offrande à la déesse ${ }^{48}$. La scène pourrait évoquer la description par Pausanias des sacrifices ancestraux accomplis par les Éléens à Olympie, qui consistait à brûler sur l'autel des rameaux d'olivier, en plus de l'encens mêlé à du blé et du miel ${ }^{49}$. Par ailleurs, le kanoun est souvent représenté dans la peinture de vase contenant des rameaux dressés, qui, dans certains cas, rappellent un élément végétal figurant ailleurs dans la scène. La signification de cette représentation est toutefois incertaine ${ }^{50}$.

19 À défaut de parallèles, on ne peut assurer l'interprétation de la procédure décrite dans le calendrier de Cos. L'olivier et le laurier avaient-ils pour vocation de remplacer les grains d'orge par des feuilles? ou bien des rameaux étaient-ils utilisés en parallèle, comme c'était peut-être le cas dans le règlement de Lykosoura mentionné plus haut, où l'olivier figure aux côtés des grains d'orge parmi les prothymata ${ }^{51}$ ? Quoi qu'il en soit, cet exemple atteste que les rites d'ouverture du sacrifice sanglant étaient probablement plus complexes et plus variés que ce que les sources littéraires permettent d'entrevoir.

\section{Conclusions}

La difficulté de cerner dans toute son ampleur la pratique du lancer d'orges en ouverture du sacrifice grec tient à la disparité des sources qui en font part. Tandis que les inscriptions sont peu explicites en la matière, chaque genre littéraire, en fonction de sa spécificité, mettra l'accent sur tel ou tel aspect du sacrifice. Il en va de même des sources iconographiques, qui ont leur langage propre. Si la représentation du sacrifice homérique diffère par bien des points de celle des époques postérieures ${ }^{52}$, il est possible d'y observer, en ce qui concerne le lancer d'orges, des caractéristiques qui seront 


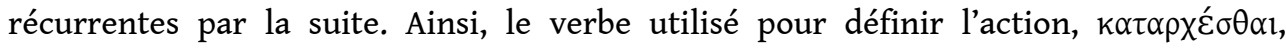
évoque l'idée de commencement, d'ouverture. De même, plutôt que la destination des grains, c'est le mouvement vers l'avant - symbolisant également une forme d'ouverture - qui est mis en évidence. Or, on a constaté que le geste était étroitement associé à la prononciation de la prière. Le lancer d'orges aurait donc pour fonction de ponctuer le début de la procédure sacrificielle et d'ouvrir la communication avec le divin en accompagnant l'invocation à la divinité. En somme, c'est le geste qui est joint à la parole. Par ailleurs, il permet également l'inclusion dans la communauté sacrifiante puisque, dans l'épopée homérique du moins, il est accompli par tous les participants ${ }^{53}$.

Une telle interprétation va à l'encontre de la nature agressive supposée du geste, qui a été perçue comme le refus d'une nourriture primordiale ou comme une mise à l'écart de l'animal, sur le point d'être mis à mort. Cette « lapidation symbolique » de l'animal, avancée notamment par W. Burkert, se fonde sur un passage de Pausanias, qui décrit un sacrifice à Térée à Mégare où les oulai étaient remplacés par des pierres ${ }^{54}$. Or, le Périégète présente ce cas comme une exception à la norme ; il ne peut donc être utilisé comme argument en faveur d'une nature intrinsèquement violente du geste, mais serait plutôt à mettre en rapport avec l'histoire du destinataire et le cas particulier de ce sacrifice ${ }^{55}$. Par ailleurs, la symbolique de l'orge, en tant que nourriture primordiale, est fréquemment exprimée dans les sources antiques. Au-delà de toute représentation culturelle, il faut remarquer que l'orge était la céréale la plus cultivée en Grèce, car elle était bien adaptée au climat, avec une résistance au manque d'humidité plus importante que le blé, et constituait donc une part importante de l'alimentation. L'interprétation du lancer d'orges comme un refus d'une période où cette céréale était encore consommée s'en trouve largement nuancée : même si les grains n'étaient plus consommés entiers, tels quels, la référence reste la même. Enfin, on ne peut nier l'existence, dans les sources, d'un certain système de représentations selon lequel la consommation de l'orge, et la culture céréalière en général, déterminerait le passage de l'état animal, qui ne connaît pas la parole, au statut d'humain, pourvu de raison; de la barbarie à la civilisation. Le sacrifice du troupeau d'Hélios dans l'odyssée, où les orges et le vin sont remplacés par des feuilles et de l'eau, comme une forme de retour vers la barbarie, en est une belle illustration. L'idée ne peut manquer d'évoquer l'interprétation de J.-P.Vernant du mythe de Prométhée chez Hésiode comme fondateur de la condition humaine, qui se définit par la procréation, la culture de la terre et l'offrande de sacrifices aux dieux ${ }^{56}$.

Le lancer d'orges en ouverture du sacrifice apparait donc comme un geste plurifonctionnel et pluri-référentiel. Si l'examen des sources épigraphiques ne permet guère de préciser le déroulement de cette pratique, ni sa signification, celles-ci suggèrent que le lancer de grains d'orge s'insérait dans le cadre plus large des prothymata, gestes accomplis en prélude à la mise à la mort de l'animal, dont la complexité et la diversité ne doit pas être sous-estimée. 


\section{BIBLIOGRAPHIE}

ACKERMANN D., « Rémunération des prêtres et déroulement des cultes dans un dème de l'Attique. Le règlement religieux d'Aixonè ", LEC 75 (2007), p. 111-136.

AUBRIOT-SÉVIN D., Prière et conceptions religieuses en Grèce ancienne jusqu'àla fin duv viècle av. J.-C., Lyon, 1992 (Collection de la Maison de l'Orient Méditerranéen no 22, Série Littéraire et Philosophique, 5).

BEDNAREK B., « Whole and Ground: krithai and alphita», in BIELAWSKI K. (éd.), Animal Sacrifice in Ancient Greece. Proceedings of the First International Workshop in Kraków (12-14.11.2015), Warzawa, 2017, p. 145-182.

BLECH M., Studien zum Kranz bei den Griechen, Berlin/New York, 1982 (Religionsgeschichtliche Versuche und Vorarbeiten, 38).

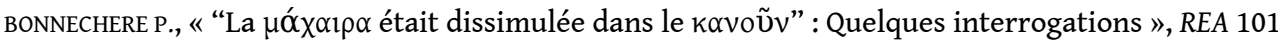
(1999), p. 21-35.

BREMMER J.N., « Greek Normative Animal Sacrifice », in OGDEN D. (ed.), A Companion to Greek Religion, Malden (MA), 2007, p. 132-144.

BRUIT ZAIDMAN L., « Offrandes et nourritures : repas des dieux et repas des hommes en Grèce ancienne », in GEORGOUDI S., KOCH PIETTRE R., SCHMIDT F. (ed.), La cuisine et l'autel. Les sacrifices en questions dans les sociétés de la Méditerranée ancienne, Turnhout, 2005 (Bibliothèque de l'École des Hautes Études, Sciences Religieuses, 124), p. 31-46.

BURKERT W., « Greek Tragedy and Sacrificial Ritual », GRBS 7 (1966), p. 87-121.

-, Homo Necans. The Anthropology of Ancient Greek Sacrificial Ritual and Myth, Berkeley et al., 1983.

CHANTRAINE P. et al., Dictionnaire étymologique de la langue grecque : histoire des mots, Paris, 1999.

CORLU A., Recherches sur les mots relatifs à l'idée de prière, d'Homère aux tragiques, Paris, 1966

(Études et commentaires, 64).

DETIENNE M., « Pratiques culinaires et esprit de sacrifice », in DETIENNE M., VERNANT J.-P. (ed.), La cuisine du sacrifice en pays grec, Paris, 1979, p. 7-35.

DURAND J.-L., Sacrifice et labour en Grèce ancienne. Essai d'anthropologie religieuse, Paris, 1986.

EKROTH G., « Pausanias and the Sacrificial Rituals of Greek Hero-cults », in HÄGG R. (ed.), Ancient Greek Hero Cult. Proceedings of the Fifth International Seminar on Ancient Greek Cult, organized by the Department of Classical Archaeology and Ancient History, Göteborg University, 21-23 April 1995, Stockholm, 1999 (Acta Instituti Atheniensis Regni Sueciae, Series in 8²,16), p. 145-158.

FOLEY H., Ritual Irony: Poetry and Sacrifice in Euripides, Ithaca, 1985.

GEORGOUDI S., « Le consentement de la victime sacrificielle : une question ouverte », in MEHL V., BRULÉ P. (ed.), Le sacrifice antique. Vestiges, procédures et stratégies, Rennes, 2007 (Collection « Histoire »), p. 139-153.

GRAF F., « Milch, Honig und Wein. Zum Verständnis der Libation im griechischen Ritual », in Perennitas. Studi in onore di Angelo Brelich promossi dalla Cattedra di Religioni del mondo classico dell'Università degli Studi di Roma, Roma, 1980, p. 209-221. 
-, « What is New about Greek Sacrifice? », in HORSTMANSHOFF H.F.J. (ed.), Kykeon. Studies in Honour of H.S. Versnel, Leiden, 2002 (Religions in the Graeco-Roman World, 142), p. 113-125.

HENRICHS A., « Animal Sacrifice in Greek Tragedy. Ritual, Metaphor, Problematizations », in FARAONE C.A., NAIDEN F.S. (ed.), Greek and Roman Animal Sacrifice. Ancient Victims, Modern Observers, Cambridge, 2012, p. 180-194.

HITCH S., King of Sacrifice. Ritual and Royal Authority in the Iliad, Washington DC, 2009 (Hellenic Studies, 25).

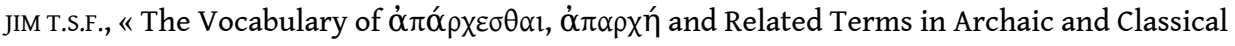
Greece ", Kernos 24 (2011), p. 39-58.

KEARNS E., " Cakes in Greek Sacrifice Regulations », in HÄGG R. (ed.), Ancient Greek Cult Practice from Epigraphical Evidence. Proceedings of the Second International Seminar on Ancient Greek Cult, organized by the Swedish Institute at Athens, 22-24 November 1991, Stockholm, 1994 (Acta Instituti Atheniensis Regni Sueciae, Series in $\left.8^{\circ}, 13\right)$, p. 65-70.

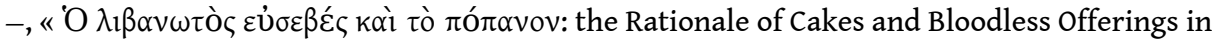
Greek Sacrifice », in PIRENNE-DELFORGE V., PRESCENDI F. (ed.), « Nourrir les dieux ?» Sacrifice et représentation du divin, Liège, 2011 (Kernos, suppl. 26), p. 89-103.

KEFALIDOU E., « Ceremonies of Athletic Victory in Ancient Greece », Nikephoros 12 (1999), p. 95-119. KIRK G.S., « Some Methodological Pitfalls in the Study of Ancient Greek Sacrifice (in Particular) », in RUDHARDT J., REVERDIN O. (ed.), Le sacrifice dans l'Antiquité, Vandœuvres/Genève, 1980 (Entretiens sur l'Antiquité classique, 27), p. 41-90.

PARKER R., On Greek Religion, Ithaca/London, 2011 (Townsend Lectures/Cornell Studies in Classical Philology, 40).

PAUL S., Cultes et sanctuaires de l'île de Cos, Liège, 2013 (Kernos, suppl. 28).

REDFIELD J., « Animal Sacrifice in Comedy. An Alternative Point of View », in FARAONE CHRISTOPHER A., NAIDEN F.s. (ed.), Greek and Roman Animal Sacrifice. Ancient Victims, Modern Observers, Cambridge, 2012, p. 167-179.

RUDHARDT J., Notions fondamentales de la pensée religieuse et actes constitutifs du culte dans la Grèce classique, Paris, $1992^{2}$ (Antiquité/Synthèses, 3).

SCHEID J., Quand faire, c'est croire. Les rites sacrificiels des Romains, Paris, 2005 (Collection historique). SCHELP J., Das Kanoun: der griechische Opferkorb, Würzburg, 1975 (Beiträge zur Archäologie, 8).

SCHWENN F., Gebet und Opfer: Studien zum griechischen Kultus, Heidelberg, 1927

(Religionswissenschaftliche Bibliothek, 8).

SEAFORD R., Reciprocity and Ritual. Homer and Tragedy in the Developing City-State, Oxford, 1994.

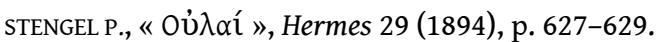

-, « Opferblut und Opfergerste », Hermes 41 (1906), p. 230-246.

-, Opferbräuche der Griechen, Leipzig/Berlin, 1910.

VAN STRATEN F.T., Hierà kalá. Images of Animal Sacrifice in Archaic and Classical Greece, Leiden, 1995 (Religions in the Graeco-Roman World, 127).

VERNANT J.-P., « Manger aux pays du Soleil », in DETIENNE M., VERNANT J.-P. (ed.), La Cuisine du sacrifice en pays grec, Paris, 1979, p. 239-249. 
-, «À la table des hommes. Mythe de fondation du sacrifice chez Hésiode », in DETIENNE M., VERNANT J.-P. (ed.), La Cuisine du sacrifice en pays grec, Paris, 1979, p. 37-132.

VIDAL-NAQUET P., Le chasseur noir. Formes de pensée et formes de société dans le monde grec, Paris, 1991

[1981] (Textes à l'appui).

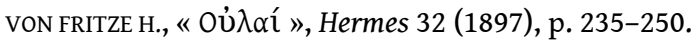

VOUTIRAS E., " Opfer für Despoina: Zur Kultsatzung des Heiligtums von Lykosura IG V 2, 514 », Chiron 29 (1999), p. 233-249.

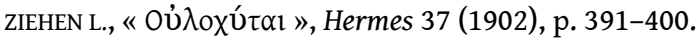

\section{NOTES}

1. Parmi les exceptions, voir récemment KEARNS (1994); KEARNS (2011); BRUIT ZAIDMAN (2005); dernièrement, BEDNAREK (2017), paru après la rédaction du présent article.

2. VON FRITZE (1897), se fondant notamment sur Théophraste, apud Porphyre, De Abst. II, 6. Voir également SCHELP (1975), p. 24, qui y voit une référence à un sacrifice ancestral, non sanglant.

3. ZIEHEN (1902).

4. STENGEL (1906), p. 243-246; STENGEL (1910), p. 13-16.

5. DETIENNE (1979), p. 12-13; 19-20 ; DURAND (1986), p. 123-133; BRUIT ZAIDMAN (2005), p. 34. Le lien avec une alimentation originelle est abondamment commenté par BEDNAREK (2017).

6. BURKERT (1983), p. 4-5; GRAF (2002), p. 121.

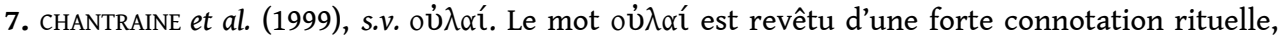

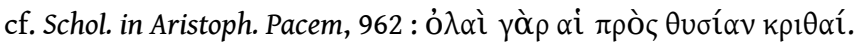

8. Il. I, 449 et 458 ; II, 410 et 421 ; Od. III, 445 et 447 . Les oulochytai sont absents de la description du sacrifice qui accompagne un serment (Il. III, 269-301), d'un sacrifice déjà en cours (Od. III, 5-66), ou du sacrifice d'Eumée (Od. XIV, 414-453). L'association des oulochytai avec la prière dans l'épopée homérique est également soulignée par BEDNAREK (2017), p. 162-164.

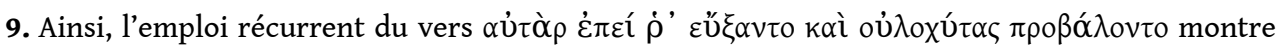
clairement l'articulation des deux actions. Quant au verbe $\alpha$ vaı $\rho \tilde{\imath} v$, il évoque l'idée de prendre les grains en main hors d'un récipient, sans doute le kanoun. Le même verbe désigne ailleurs le fait de soulever l'animal sacrificiel de terre (Od. III, 453).

10. XII, 356-363. Voir infra.

11. IV, 758-767 (trad. V. Bérard, CUF).

12. ZIEHEN (1902).

13. Par exemple AUBRIOT-SÉVIN (1992), p. 104, n. 275 ; CORLU (1966), p. 89-91. Selon SCHWENN (1927), p. 37 ; SCHELP (1975), p. 14, l'offrande est le substitut d'un sacrifice que Pénélope ne serait pas en mesure d'accomplir.

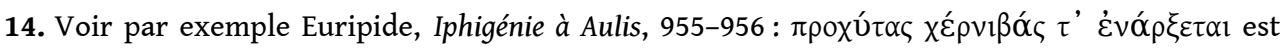
synonyme de «faire le sacrifice». Sur l'iconographie, voir DURAND (1986), p. 123-133 et VAN STRATEN (1995), p. 31-43.

15. Straton, Phoenicides (= Athénée, IX, 383), montre que le terme était sans doute tombé en désuétude dans le langage courant (voir infra). Hormis les scholies à Homère, on trouve encore le terme dans des œuvres épiques d'inspiration homérique : Apollonios de Rhodes, I, 409 ; Quintus

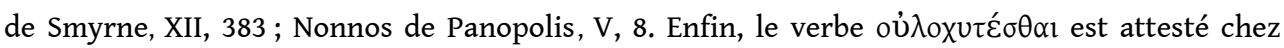
Théophraste (voir infra, n. 32).

16. Aristophane, Paix, 948-966. 


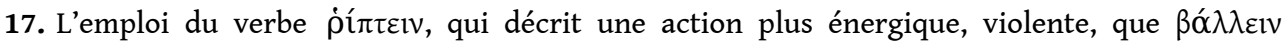
(CHANTRAine et al. [1999], s.v. pín $\tau \omega$ ), pourrait aller dans le même sens. Par l'emploi du mot $\theta \varepsilon \alpha \tau \alpha \tilde{\imath} \varsigma$, qui fait clairement référence aux spectateurs de la pièce, Aristophane joue de plus sur les deux registres de la représentation et du temps présent. Sur ce passage, voir également VAN STRATEN (1995), p. 38-39, qui évoque un possible parallèle dans la peinture de vase.

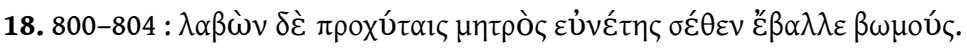

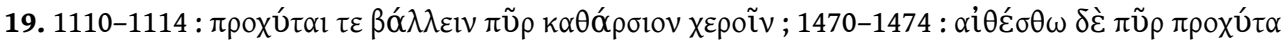
ı५ $\kappa \alpha \theta \alpha \rho \sigma i ́ o ı \sigma$. L'hypallage paraît être confirmé par d'autres occurrences du feu purificateur chez le même auteur (Héraclès, 937 ; Hélène, 869 ; Électre, 812).

20. Sur la tragédie, voir BURKERT (1966) ; HENRICHS (2012). Sur la comédie, voir REDFIELD (2012) ; sur le caractère improvisé du sacrifice, voir Ménandre, Dyscolos, 393-521; Samienne, 222-223 ; La Tondue, 996-998. Cf. BONNECHERE (1999), p. 26.

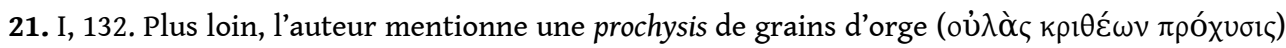
qui semble rendre compte du même geste, lorsqu'il évoque que les habitants de chios ont un scrupule à utiliser la production agricole du territoire d'Atarnée en Mysie qui a été acquis de manière impie, à savoir en échange d'un suppliant livré aux Perses.

22. VII, 297d. Voir également Dion Chrysostome 31, 10, 8.

23. Selon DURAND (1986), p. 124, le lancer d'orges participerait à l'assentiment de l'animal, mais la notion de consentement de la victime sacrificielle a été remise en question récemment, voir GEORGOUDI (2007).

24. FOLEY (1985), p. 31 ; SEAFORD (1994), p. 45.

25. V, 6-8.

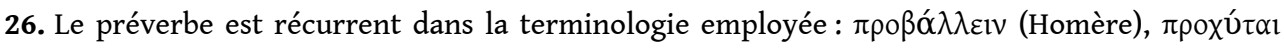

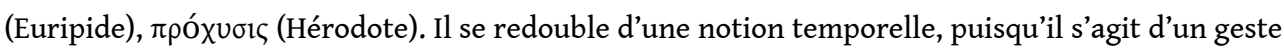
accompli avant la mise à mort.

27. Voir à ce propos la note de STENGEL (1894). L'idée revient de manière récurrente dans les commentaires et les traductions, notamment RUDHARDT (1992²), p. 254 et 259; DETIENNE (1979), p. 19 ; BREMMER (2007), p. 136 ; PARKER (2011), p. 153.

28. Straton, Phoenicides (= Athénée, IX, 383).

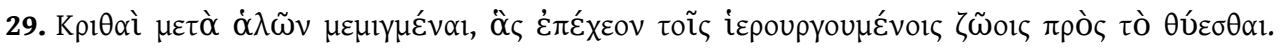
Mélange des orges et du sel : Orion, Etymologicum, s.v. oủ

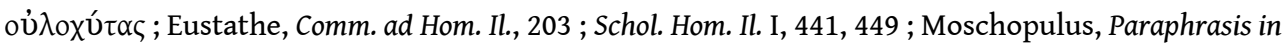

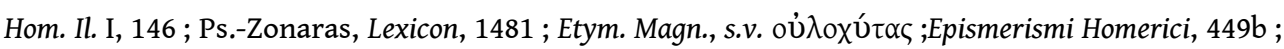
Tzetses, 99. Le lancer de grains vers l'animal: Schol. Hom. Il. I, 441, 449 ; Epismerismi Homerici, 449b ; Ps.-Zonaras, Lexicon, 1481. Voir BEDNAREK (2017), pour un commentaire plus détaillé de ces sources.

30. SCHEID (2005), p. 50-55, selon lequel l'immolatio symbolise les deux parties concernées, à savoir les céréales pour les humains et le vin pour la sphère divine. La comparaison entre les deux pratiques grecque et romaine est établie explicitement par Denys d'Halicarnasse, II, 25 ; VII, 72, arguant que les Grecs utilisent de l'orge pour commencer les sacrifices parce qu'ils la considèrent comme plus ancienne, tandis qu'à Rome, c'est l'épeautre qui tient ce rôle.

31. Théophraste, apud Porphyre, De Abstinentia II, 6 ; Plutarque, Questions grecques, 292c ; Denys d'Halicarnasse, II, 25.

32. Sur l'offrande de gâteaux, voir KEARNS (1994); KEARNS (2011); BRUIT ZAIDMAN (2005). Un règlement cultuel du dème d'Aixonè, en Attique ( $1^{\text {re }}$ moitié $d u \mathrm{IV}^{\mathrm{e}} \mathrm{s}$.) montre bien l'importance de ces offrandes supplémentaires, pour la provision desquelles le prêtre obtenait une compensation en argent : CGRN 57. Cf. ACKERMANN (2007).

33. KEARNS (1994), p. 69. 
34. Une possible exception vient d'une inscription de Naxos (IG XII 5, 45) selon laquelle

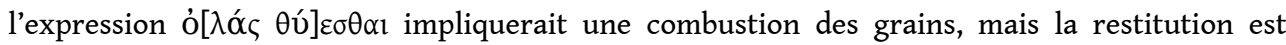
importante.

35. LSS 22 / CGRN 64 (fin IV s.).

36. LSAM 37, 1. 10-12; EA 44 (2011) : 1-54, 1. 11-13, toutes deux datées du II $\mathrm{e}$.

37. LSCG 96 / CGRN 156 (c. 200), 1.17-18. Cette mention, unique dans le calendrier, doit peut-être sa présence au fait que ce sont les hiéropes qui sont chargés de leur provision, et non le prêtre comme il est plus coutumier.

38. IG XII 4, 276 (mi-IV s.), l. 5.

39. LSCG 68 / CGRN 126 (fin $\mathrm{III}^{\mathrm{e}}$ s.), 1.13-15. Je suis ici la lecture, plus convaincante, de vouTIRAS (1999). Sur la fonction de crithologue, chargé de trier les grains d'orge, voir Plutarque, Questions grecques, 292e.

40. IG XII 4, 278 / CGRN 86 (mil. IV s.), 1. 31-33. Sur ce texte, voir PAUL (2013), passim.

41. Sur l'emploi de ce verbe, voir JIM (2011), p. 50-53.

42. PARKER (2011), p. 134.

43. GRAF (1980), p. 210. Sur la phyllobolia, voir KEFALIDOU (1999).

44. XII, 356-363.

45. Pour un commentaire de ce passage, voir VERNANT (1979) ; VIDAL-NAQUET (1991 [1981]), p. 55.

46. Sur la fonction de cet holocauste, voir PAUL (2013), p. 355-356.

47. Les scènes montrant des thallophoroi, personnages portant des branches en procession, sont particulièrement nombreuses. Sur l'utilisation de branchages dans le culte, voir BLECH (1982), p. 269-302, part. 295-297.

48. Berlin, Staatliche Museen F1686 (c. 550-540) ; VAN STRATEN (1995), p. 15 ; fig. 4 (V21).

49. $\mathrm{V}, 15,10$.

50. Il s'agit toujours de trois rameaux dressés dans le kanoun, qui pourraient servir de marque distinctive, lorsque celui-ci n'en a pas la forme typique: VAN STRATEN (1995), p.10-11. Voir notamment le cratère en cloche à figures rouges du Peintre de Kadmos (Vienne, Kunsthistorisches Museum 1144 = VAN STRATEN [1995], V371, fig. 38), où les brins du kanoun redoublent un arbuste figuré à droite de la scène. Sur la typologie et la fonction du kanoun, qui pouvait contenir diverses offrandes en plus des grains et du couteau sacrificiel, voir SCHELP (1975) ; VAN STRATEN (1995), p. 162-164.

51. LSCG 68.

52. KIRK (1980), p. 62-68; нITCH (2009), p. 19-39.

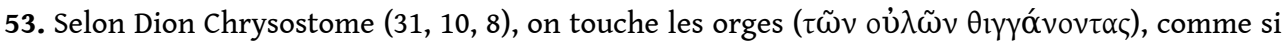
le simple fait de les manipuler déterminait l'appartenance à cette communauté. Cf. PARKER (2011), p. 151. L'assertion est également valable pour l'eau lustrale.

54. I, 48, 8-9. Cf. BURKERT (1983), p. 183.

55. Voir à ce propos ЕКROTH (1999), p. 155 et n. 38. Elle y suggère que les pierres pourraient avoir eu une fonction purificatoire, mais on pourrait également émettre l'hypothèse qu'elles étaient dues au caractère étranger $\mathrm{du}$ destinataire, pour lequel on refusait d'utiliser une céréale typiquement grecque.

56. VERNANT (1979). 


\section{RÉSUMÉS}

De l'offrande de grains à la préparation de gâteaux, les céréales font partie intégrante de la pratique sacrificielle en Grèce ancienne. Parmi leurs diverses utilisations, le lancer de grains d'orge en prélude à la mise à mort de l'animal sacrificiel a davantage attiré l'attention des commentateurs, qui en ont proposé des interprétations très diverses, se fondant tour à tour sur la symbolique de l'orge, la nature du geste ou sa place dans le rituel. Le présent article propose de faire le point sur la question en reprenant le dossier des sources littéraires, afin de réévaluer la pertinence des interprétations proposées. Dans un second temps, la documentation épigraphique, plus rarement invoquée à l'appui de cette question, permettra d'interroger le caractère immuable de cette pratique.

From offerings of grain to the baking of cakes, cereals formed an integral part of sacrificial practices in ancient Greece. Among their diverse uses, the throwing of barley grains prior to the killing of the sacrificial animal has been a point of interest. This practice has been subjected to various interpretations dependant on the symbolism of the barley, the nature of the gesture, and its position in the ritual. This paper aims to review the literary sources and re-evaluate the relevance of modern interpretations. Secondly, the epigraphic evidence, which has rarely been examined in this context, will allow us to question the possible variations within this practice.

\section{AUTEUR}

STÉPHANIE PAUL

Université de Liège

s.paul@uliege.be 